\title{
Manuscripts Cited
}

The manuscripts cited below are those to which reference is made in abbreviated form in the present volume. A complete listing and a bibliography may be found in Linker 1979, 23-69. See also Jeanroy 1918; Spanke 1955; and Aubrey 2001. For sources that preserve polyphonic music, see Ludwig 1910, 1964-78; Gennrich 1957; Reaney 1966; Everist 1989; and Van der Werf 1989.

TROUVÈRE MANUSCRIPTS

$A \quad$ Arras, Bibliothèque municipale, 657

C Bern, Burgerbibliothek, 389

$F \quad$ London, British Library, Egerton 274

$H$ Modena, Biblioteca estense, $\mathrm{R}_{4}, 4$

$I \quad$ Oxford, Bodleian Library, Douce 308

$K \quad$ Paris, Bibliothèque de l'Arsenal, 5198 ("Chansonnier de l'Arsenal”)

$L \quad$ Paris, Bibliothèque nationale de France, fr. 765

$M$ Paris, Bibliothèque nationale de France, fr. 844 ("Chansonnier du Roi," song corpus)

$N \quad$ Paris, Bibliothèque nationale de France, fr. 845

$O$ Paris, Bibliothèque nationale de France, fr. 846 ("Chansonnier Cangé”)

$P \quad$ Paris, Bibliothèque nationale de France, fr. 847

$R \quad$ Paris, Bibliothèque nationale de France, fr. I59I

$T$ Paris, Bibliothèque nationale de France, fr. I26Is ("Chansonnier de Noailles," song corpus)

$U$ Paris, Bibliothèque nationale de France, fr. 20050 ("Chansonnier de Saint-Germain-des-Prés")

$V \quad$ Paris, Bibliothèque nationale de France, fr. 24406

$X \quad$ Paris, Bibliothèque nationale de France, n. a. fr. I050 ("Chansonnier Clairambault")

$Z \quad$ Siena, Biblioteca comunale degli Intronati, H. X. 36

a Rome, Biblioteca Apostolica Vaticana, Reg. lat. 1490 
Cl Paris, Bibliothèque nationale de France, n. a. fr. I352I ("La Clayette")

F Florence, Biblioteca Medicea Laurenziana, Pluteus 29, I

Mo Montpellier, Bibliothèque interuniversitaire, Section Médecine, H 196

MüA Munich, Bayerische Staatsbibliothek, gallo-rom. 42 and Berlin, Staatsbibliothek zu Berlin, Musikabteilung 55 MS I4 (Johannes Wolf fragments)

$N$ Paris, Bibliothèque nationale de France, fr. I26Is ("Chansonnier de Noailles," motet corpus)

$R \quad$ Paris, Bibliothèque nationale de France, fr. 844 ("Chansonnier du Roi," motet corpus)

$W_{2} \quad$ Wolfenbüttel, Herzog August Bibliothek, Helmstedt I099 (Heinemann I206) 